\title{
Aporte de la hojarasca al ciclo biogeoquímico en plantaciones de Eucalyptus nitens, X Región, Chile
}

\author{
Litter contribution to biogeochemical cycle in Eucalyptus nitens plantations, X Region, Chile
}

\author{
Juan E. Schlatter ${ }^{1 *}$, Víctor Gerding ${ }^{1}$, Sigrid Calderón ${ }^{2}$ \\ *Autor de correspondencia: ${ }^{1}$ Universidad Austral de Chile, Facultad de Ciencias Forestales, \\ casilla 567, Valdivia, Chile. Fono-fax: 56-63-221431, jschlatt@uach.cl. \\ ${ }^{2}$ Corporación Chilena de la Madera, Concepción, Chile.
}

\begin{abstract}
SUMMARY
The litterfall in Eucalyptus nitens plantations, established on an andisol (close to Fresia, X Region, Chile), were measured after canopy closure (4-7 years old), in an area under different fertilization treatments. At canopy closure annual litterfall was the highest (7-11 $\left.\mathrm{tha}^{-1}\right)$, and becoming lower after it $\left(5-6 \mathrm{t} \mathrm{ha}^{-1}\right)$; the main quantity was measured in summer $(45-60 \%)$. With the annual biomass the following amounts of nutrients return to the soil $\left(\mathrm{kg} \mathrm{ha}^{-1} \mathrm{year}^{-1}\right)$ : 20-50 N, 1-4 P, 5-40 K and 31-151 Ca, respectively, depending on age and fertilizer treatment. The $\mathrm{O}$ horizon (litter layer) accumulated 6-10 t ha ${ }^{-1}$ dry matter at age 7 and the thinning and pruning, carried out at age 5-6, contributed with similar amounts of branches and bark. The litter layer accumulated $100-190 \mathrm{~N},<15 \mathrm{P}, 20-40 \mathrm{~K}$ and 140-230 $\mathrm{Ca} \mathrm{kg} \mathrm{ha}^{-1}$, respectively. This biomass and nutrients constitute an important reservoir for the ecosystem and the sustainability of future rotations.
\end{abstract}

Key words: Eucalyptus nitens, litter fall, litter layer, nutrient dynamic, biomass.

\section{RESUMEN}

El presente trabajo evalúa el flujo de hojarasca en una plantación de Eucalyptus nitens establecida en un andisol (comuna de Fresia, X Región, Chile), en un área con diferentes tratamientos de fertilización, desde el cierre de copas hasta el inicio de la edad fustal (4-7 años de edad). Al cierre de copas se alcanzó la máxima caída de hojarasca (7-11 t ha ${ }^{-1}$ ), siendo menor en los años siguientes (5-6 t ha-1); la mayor proporción (45-60\%) cayó en verano. Con esta biomasa regresaron al suelo 20-50 kg ha ${ }^{-1}$ año-1 de N, 1-4 de P, 5-40 de K y 31-151 de Ca, según edad y el tratamiento aplicado. En el mantillo se acumularon a los 7 años 6-10 t ha $\mathrm{a}^{-1}$ de biomasa y la poda y el raleo (5-6 años) adicionaron similares cantidades (ramas y corteza). En el mantillo se acumularon 90-100 kg ha-1 de N, < 15 de P, 20-40 de K y 140-230 de Ca. Esta biomasa y nutrientes constituyen una reserva muy importante para el ecosistema y la sustentabilidad del cultivo.

Palabras clave: Eucalyptus nitens, hojarasca, mantillo, dinámica nutritiva, biomasa.

\section{INTRODUCCIÓN}

Eucalyptus nitens Maiden está entre las especies de más rápido crecimiento juvenil, con culminación del crecimiento anual de la biomasa entre los 3 y 4 años de edad en plantaciones cultivadas de la X Región (Leiva 2000, Staub 2000, Aparicio 2001, Bonomelli et al. 2002), momento coincidente con el máximo incremento anual en altura y con el cierre del dosel de copas. Durante esta dinámica, los árboles en competencia recurren a los elementos nutritivos acumulados en su biomasa para cubrir parte de su demanda, ya que el suelo no puede abastecerlos en esta etapa de alta exigencia. La extracción de elementos nutritivos desde las hojas y ramas más antiguas, para abastecer el crecimiento, acelera la muerte de estos tejidos de la copa baja, lo que se traduce en caída de hojas, dando origen a la hojarasca.
La caída de hojarasca representa el mayor proceso de transferencia de nutrientes de las partes aéreas hacia el suelo (Vitousek et al. 1994) y el conocimiento del aporte en elementos nutritivos de la hojarasca es útil para orientar el manejo nutritivo de las plantaciones de Eucalyptus sp., siendo escasos los estudios en plantaciones con especies de este género (Moroni y Smethurst 2003). La hojarasca que cae al suelo forma un estrato orgánico conocido como mantillo (Schlatter et al. 2003). Este estrato cubre el suelo y lo protege de los cambios de temperatura y de humedad, y también retorna elementos nutritivos en una cantidad importante. Por descomposición y mineralización de las sustancias orgánicas los elementos nutritivos son liberados a la solución del suelo y constituyen un abastecimiento para los propios árboles, además de servir de alimento a la fauna y flora heterótrofa. 
Swift et al. (1979) señalan que las tasas de descomposición del mantillo están reguladas por tres grupos de variables: factores climáticos (fundamentalmente humedad y temperatura), la composición química del mantillo (calidad del recurso) y el tipo y cantidad de organismos (invertebrados y microorganismos). La calidad de la hojarasca afecta la abundancia, composición y actividad de la comunidad descomponedora, por lo que es el factor principal que controla las tasas de descomposición de la materia orgánica y la liberación de nutrientes (Blair et al. 1990). La calidad del mantillo se define como su relativa descomponibilidad y depende de la mezcla de compuestos lábiles, recalcitrantes e inhibitorios (Vitousek et al. 1994); es una expresión del grado en que los constituyentes químicos optimizan los requerimientos nutricionales de los saprófitos y, por lo tanto, un factor clave que determina las tasas de descomposición (Anderson 1991).

El presente trabajo tiene por objetivo general cuantificar el flujo de la hojarasca en plantaciones de Eucalyptus nitens, desde el cierre de copas en la etapa latizal hasta el inicio de la etapa fustal o de madurez del bosque, durante cuatro años. Los objetivos específicos son: a) cuantificar la biomasa y los elementos nutritivos nitrógeno, fósforo, potasio y calcio que retorna la hojarasca al suelo en el transcurso de esta etapa de la plantación; b) evaluar la variación cuantitativa de este flujo de nutrientes a lo largo del año y cómo influye en las características del mantillo; c) analizar en qué forma una intervención silvicultural (raleo) afecta el aporte de la hojarasca y al mantillo, y cómo se relaciona con la dinámica nutritiva en una plantación.

\section{MÉTODOS}

Ubicación y descripción del área. El área de estudio se ubicó en el predio Pichimaule, en la comuna de Fresia $\left(41^{\circ} 01^{\prime} \mathrm{S}-73^{\circ} 27^{\prime} \mathrm{O}\right)$, en una altitud de $200 \mathrm{~m}$ s.n.m. en lomajes de transición entre la Depresión Intermedia y la Cordillera de la Costa, con pendientes suaves (5-10\%). El suelo corresponde a la serie Crucero (Acrudoxic Hydric Hapludand) cuyo material parental pertenece a cenizas volcánicas, probablemente pleistocénicas, las que cubren sedimentos fluvioglaciales, dando origen a suelos con textura franca a arcillosa sobre antiguas terrazas disectadas (CIREN 2001). El suelo es homogéneo en toda el área destinada al estudio: profundo, con buena estructura y drenaje interno moderado a lento en el subsuelo; con anterioridad a la plantación forestal hubo ganadería extensiva en pradera natural y un historial agrícola.

En el perfil de suelo se identificaron los horizontes A $(0-6 \mathrm{~cm}), \mathrm{AB}(6-17 \mathrm{~cm}), \mathrm{BA} 1(17-27 \mathrm{~cm}), \mathrm{BA} 2(27-40 \mathrm{~cm})$, $\mathrm{B}(\mathrm{g}) 1(40-67), \mathrm{B}(\mathrm{g}) 2(67-90 \mathrm{~cm})$ y $\mathrm{Bg}(90->110 \mathrm{~cm})$. En el estrato $0-27 \mathrm{~cm}$ la textura fue franca y los colores predominantes fueron 5YR 3/1-3/2; a mayor profundidad la textura fue franca arenosa y los colores variaron de
5YR 3/2 a 5YR 4/3. El contenido de materia orgánica era de $12,4 \%$ hasta los $17 \mathrm{~cm}$ de profundidad, $4,5 \%$ en $27-67 \mathrm{~cm}$ y $2,6 \%$ en $67-110 \mathrm{~cm}$ de profundidad. El pH en agua fue de 5,1, 4,6 y 5,1 respectivamente. El contenido de fósforo disponible (Olsen) varió de 2 a 4 mg kg-1, en profundidad.

De acuerdo a la clasificación Koeppen, el clima es de costa occidental con influencia mediterránea (Donoso 1992). Existe una frecuencia de heladas de 20-30 días por año, con un periodo libre de heladas de 150-200 días por año y una temperatura mínima de $-6^{\circ} \mathrm{C}$. El régimen de humedad anual es moderado, con una precipitación de 1.600-2.000 mm anuales, lo que marca un excedente para el consumo de la vegetación, no obstante en verano aumenta la evapotranspiración, por lo cual se presenta un déficit hídrico (Schlatter et al. 1995).

Características de la plantación. La plantación forestal se realizó en junio de 1996 con 1.666 plantas ha ${ }^{-1}$ (4 m $\mathrm{x} 1,5 \mathrm{~m}$ ). Las plantas, producidas en contenedores con semilla de Australia (Macalyster, Victoria Central), se seleccionaron por altura $(25-30 \mathrm{~cm})$ y diámetro de cuello (4 mm). Previo a la plantación se realizó un control químico de malezas y un subsolado de $50 \mathrm{~cm}$ de profundidad en las líneas. Posterior a la plantación se realizaron algunos tratamientos de fertilización y controles químico y manual de malezas (Leiva 2000, Staub 2000, Aparicio 2001, Gerding et al. 2002, Belmar 2003, González 2003). De esos tratamientos sólo cuatro fueron considerados para este trabajo, con el fin de responder a los objetivos aquí planteados. No es objetivo de este trabajo identificar el efecto de la fertilización, por lo cual las unidades de manejo nutritivo distinto fueron simplemente diferenciados como rodales. En el cuadro 1 se identifican y resumen las características de los rodales seleccionados, indicando en cuáles fueron realizados raleo y poda. Estas intervenciones silviculturales se efectuaron a mediados del año 2001; a los 5 años de edad, se extrajo sólo la madera luego del desrame y descortezado in situ (los desechos quedaron esparcidos sobre el suelo); la poda alcanzó a 4 m de altura. En el cuadro 2 se describen las plantaciones a los 7 años de edad (al final del período de evaluación). Los tres rodales con raleo tuvieron similar resultado en biomasa al séptimo año; en cambio, aquel sin raleo acumuló más biomasa aérea como era de esperar.

Diseño experimental. En agosto de 1999 se instalaron en cada rodal, en un área de 50 x 50 m, seis cajones recolectores de hojarasca de $0,5 \times 0,5 \mathrm{~m}$, distribuidos en dos grupos de tres cajones. Cada grupo se dispuso de tal forma, que un recolector estuviera en la hilera de plantación (posición A), otro en el punto medio entre las hileras (posición C) y un tercero equidistante entre ellos (posición B), para así considerar las variaciones de cobertura. La hojarasca se comenzó a recibir desde el 1 de septiembre de 1999, con recolección mensual. Las muestras se 
Cuadro 1. Características de los tratamientos de fertilización seleccionados en Eucalypus nitens, predio Pichimaule, comuna de Fresia, provincia de Llanquihue, X Región.

Selected fertilization treatments in the experimental area.

\begin{tabular}{|c|c|c|c|c|c|}
\hline $\begin{array}{c}\text { Aplicación } \\
\text { preplantación } \\
\text { (1996) }\end{array}$ & $\begin{array}{c}\text { Fertilización } \\
\text { posplantación } 1 \text { (oct-96) }\end{array}$ & $\begin{array}{c}\text { Fertilización } \\
\text { posplantación } 2 \text { (sep-97) }\end{array}$ & $\begin{array}{c}\text { Fertilización } \\
\text { posplantación } 3 \text { (ago-99) }\end{array}$ & Raleo & Rodal \\
\hline \multirow[b]{2}{*}{ Testigo } & Sin fertilización & Sin fertilización & Sin fertilización & No & 0 \\
\hline & $\begin{array}{l}135 \text { g planta }^{-1} \text { de } \\
\text { NPK }(20: 25: 5)+ \\
\text { micronutrientes }\end{array}$ & $\begin{array}{c}96 \mathrm{~kg} \mathrm{ha}^{-1} \mathrm{P}, 45 \mathrm{~N}, 10 \mathrm{~K} \\
\text { B, Cu y Zn }\end{array}$ & $\begin{array}{l}112 \mathrm{~kg} \mathrm{ha}^{-1} \mathrm{P} \mathrm{y} \\
230 \mathrm{~kg} \mathrm{ha}^{-1} \mathrm{~N}\end{array}$ & Sí & 1 \\
\hline \multirow{2}{*}{$\begin{array}{c}\text { Bifox } \\
48 \mathrm{~kg} \mathrm{ha}^{-1} \text { de } \mathrm{P}\end{array}$} & \multirow{2}{*}{$\begin{array}{l}135 \mathrm{~g}_{\text {planta }}{ }^{-1} \mathrm{de} \\
\text { NPK }(20: 25: 5)+ \\
\text { micronutrientes }\end{array}$} & \multirow{2}{*}{$\begin{array}{c}48 \mathrm{~kg} \mathrm{ha}^{-1} \mathrm{P}, 45 \mathrm{~N}, 10 \mathrm{~K} \\
\mathrm{~B}, \mathrm{Cu} \text { y } \mathrm{Zn}\end{array}$} & Sin fertilización & Sí & 2 \\
\hline & & & $\begin{array}{l}100 \mathrm{~kg} \mathrm{ha}^{-1} \mathrm{P} \mathrm{y} \\
230 \mathrm{~kg} \mathrm{ha}^{-1} \mathrm{~N}\end{array}$ & Sí & 3 \\
\hline
\end{tabular}

Cuadro 2. Características de las plantaciones de Eucalypus nitens a los 7 años de edad, predio Pichimaule, Fresia, X Región.

Characteristics of the E. nitens plantations at 7 years old according to treatment, Fresia, $10^{\text {th }}$ Region.

\begin{tabular}{ccccc}
\hline Rodal & $\begin{array}{c}\text { DAP medio } \\
(\mathrm{cm})\end{array}$ & $\begin{array}{c}\text { Altura } \\
\text { dominante } \\
(\mathrm{m})\end{array}$ & $\begin{array}{c}\text { Biomasa } \\
\text { aérea } \\
\left(\mathrm{t} \mathrm{ha}^{-1}\right)\end{array}$ & $\begin{array}{c}\text { Biomasa aérea } \\
\text { cortada } \\
\text { con el raleo } \\
\left(\mathrm{t} \mathrm{ha}^{-1}\right)\end{array}$ \\
\hline $0 *$ & 19 & 21,8 & 167 & 0 \\
1 & 21 & 22,8 & 128 & 59 \\
2 & 21 & 24,0 & 117 & 59 \\
3 & 20 & 23,7 & 112 & 55
\end{tabular}

* No fue raleado y tenía densidad de 1.500 árboles ha ${ }^{-1}$; los otros rodales tenían densidad de 800 árboles ha ${ }^{-1}$ después del raleo.

Not thinned with 1.500 trees $\mathrm{ha}^{-1}$; others thinned to 800 trees $^{-1} \mathrm{a}^{-1}$

pesaron individualmente (secado en estufa $75^{\circ} \mathrm{C}$ ). En el año 2001 (junio) se amplió el número de cajones recolectores a nueve por rodal, formando tres grupos distribuidos al azar, de tres cajones cada uno, procediendo en el resto del método de la misma forma ya descrita.

La cantidad de hojarasca recolectada y pesada por cajón fue posteriormente mezclada por cada grupo de cajones y por estación del año, correspondiendo a primavera: septiembre, octubre y noviembre; verano: diciembre, enero y febrero; otoño: marzo, abril y mayo, e invierno: junio, julio y agosto. Estas muestras mezcladas fueron pulverizadas en molino y analizadas en su contenido de: nitrógeno (Kjeldahl), fósforo (calcinación, $\mathrm{HCl}$ al $10 \%$ y colorometría), potasio y calcio (calcinación, $\mathrm{HCl}-10 \%$ y lectura con espectrometría de absorción atómica) ${ }^{1}$.

1 Laboratorio de Nutrición y Suelos Forestales, Facultad de Ciencias Forestales, UACh.
En mayo del año 2000 se recolectó mantillo en cada rodal, en tres grupos ubicados al azar y en cada grupo se obtuvieron tres muestras de $0,1 \mathrm{~m}^{2}$ ubicadas en las posiciones A, B y C antes descritas. En estas muestras se determinó en terreno el espesor total del mantillo (horizonte $\mathrm{O}$ ), hasta el suelo mineral. El procedimiento anterior se repitió entre marzo y abril de los años 2001, 2002 y 2003. El material del mantillo fue separado en los componentes de Oi y Oe (el subhorizonte Oa no se presentó) para los años 2002 y 2003 y, además, ramas y corteza, para identificar el efecto del raleo realizado a mediados del año 2001. De todos ellos se obtuvo el peso seco $\left(75^{\circ} \mathrm{C}\right)$ en cada muestra. El mantillo y/o sus componentes se mezclaron por grupo y luego se procedió a molerlos (molino) y a su análisis químico de la misma forma que para la hojarasca, obteniendo tres muestras mezcla por rodal en cada oportunidad de muestreo.

El análisis de datos se realizó a través de estadística descriptiva, análisis de varianza y multicomparación de medias de Scheffé, regresión lineal y correlación de Pearson (Hartung et al. 2002).

\section{RESULTADOS}

Como ejemplo del modelo de variación temporal de la caída mensual de hojarasca se presenta lo observado en el rodal 0 (figura 1). En este rodal casi no hubo caída de hojarasca antes del inicio de las mediciones, por lo que el histograma refleja el proceso desde su inicio. Las cantidades mayores se presentaron en noviembre y diciembre del año 1999, con valores de 3.585 y $2.176 \mathrm{~kg} \mathrm{ha}^{-1}$, respectivamente, como respuesta al cierre de copas. Desde entonces la mayor cantidad de aporte mensual fue en enero, es decir, durante el verano, con valores de $945,1.946$ y $1.614 \mathrm{~kg} \mathrm{ha}^{-1}$ para los años 2001 , 2002 y 2003, respectivamente. La menor caída de desechos ocurrió en los meses de mayo y junio de cada año. 
La variación estacional de la caída de la hojarasca en los cuatro rodales se muestra en la figura 2. El rodal 3 aparece con la menor cantidad de hojarasca al inicio (2.129 $\mathrm{kg} \mathrm{ha}^{-1}$ en primavera de 1999) con respecto a los demás rodales, debido a un cierre prematuro de sus copas, ya que en este rodal la plantación creció inicialmente más rápida, por la fertilización que tuvo, cayendo la mayor cantidad de hojarasca un período vegetativo antes. Todos los rodales mostraron, sin embargo, la misma tendencia de variación. La diferencia cuantitativa que se aprecia entre el rodal 0 y los tres restantes, a partir de la primavera de 2001, se debe al raleo al que estos últimos fueron sometidos.

Las cantidades anuales de hojarasca mostradas en la figura 3 incluyen estimaciones del período previo a lo medido, basadas en los resultados de Bonomelli et al. (2002) para los primeros tres años de crecimiento, pero ajustados a la dinámica de crecimiento del sitio. El ro- dal 3 presentó la mayor cantidad de hojarasca en el período 1998-1999, por lo anteriormente explicado. Efectivamente, en los rodales 1 y 3 ya había bastante hojarasca acumulada en el suelo bajo los árboles (línea de plantación) al iniciarse las mediciones. No así en los rodales 0 y 2 , cuyas copas cerraron posteriormente.

En la figura 4 se observa la cantidad de mantillo acumulado en cada tratamiento desde los 4 hasta los 7 años de edad. La mayor cantidad de mantillo en los años 2002 y 2003 (6 y 7 años) en los rodales con raleo fue producto de ramas, despuntes y corteza depositados en el sitio por esa intervención silvicultural. El rodal 0 mostró un leve ascenso en la cantidad de mantillo al incorporar material de ramas y corteza caído naturalmente desde el año 2002. La mayor acumulación de horizonte $\mathrm{O}(P<0,05)$ en los rodales 1 y 3 en el año 2000, con respecto al año 2001, tiene su origen en lo indicado en la figura 3, sobre el cierre de copas previo en estos rodales.

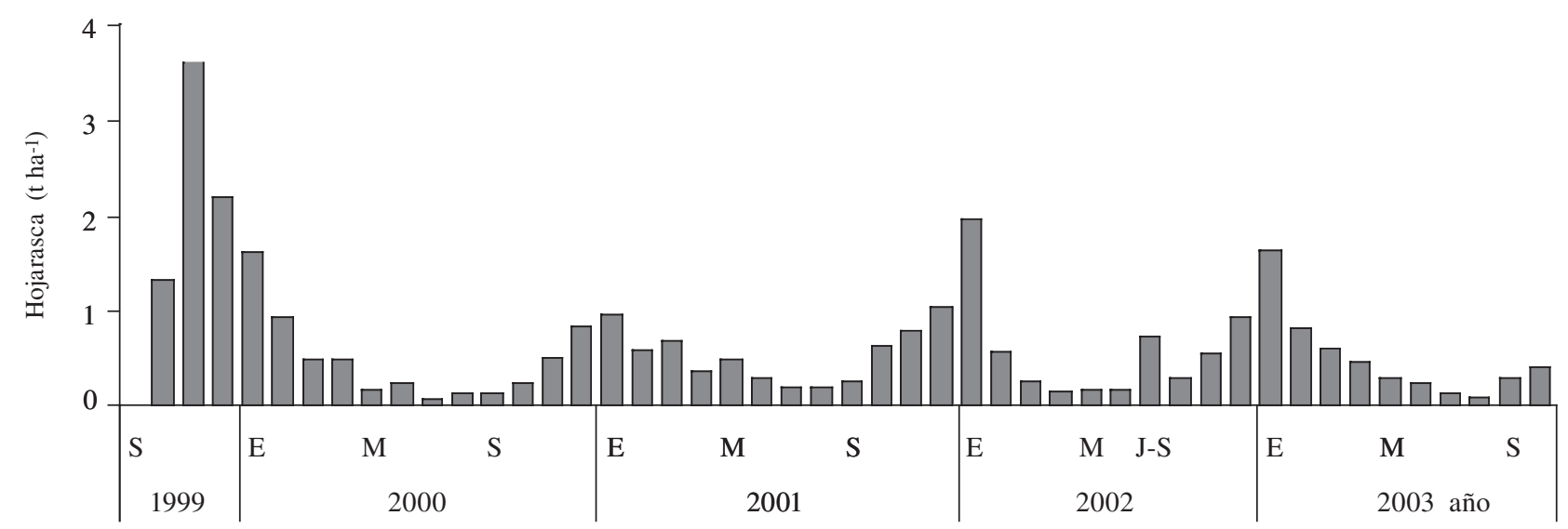

Figura 1. Aporte mensual de hojarasca para el período 1999-2003, en Eucalyptus nitens, rodal 0, predio Pichimaule, Fresia, X Región.

Monthly litter fall between 1999 and 2003 in E. nitens, stand 0, Fresia, $10^{\text {th }}$ Region.

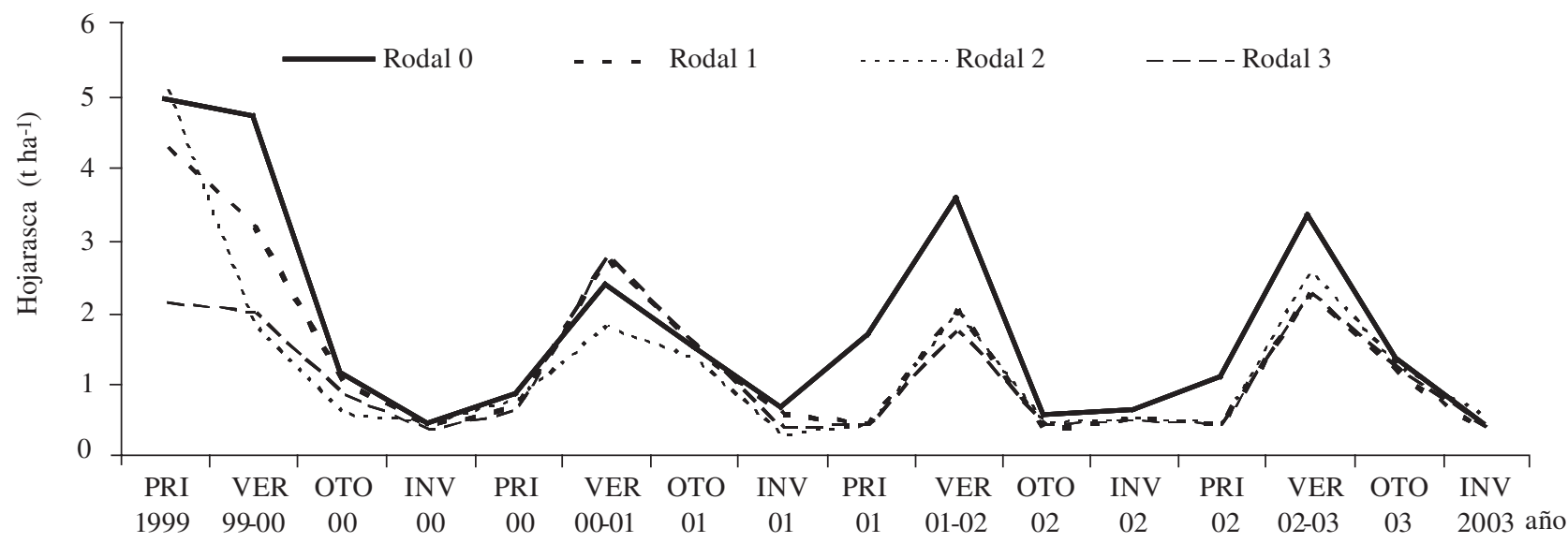

Figura 2. Variación estacional del aporte de hojarasca para cuatro rodales en Eucalyptus nitens (rodal 0: no raleado), predio Pichimaule, Fresia, X Región.

Seasonal variation of litter fall in four stands of E. nitens, Fresia, $10^{\text {th }}$ Region. 


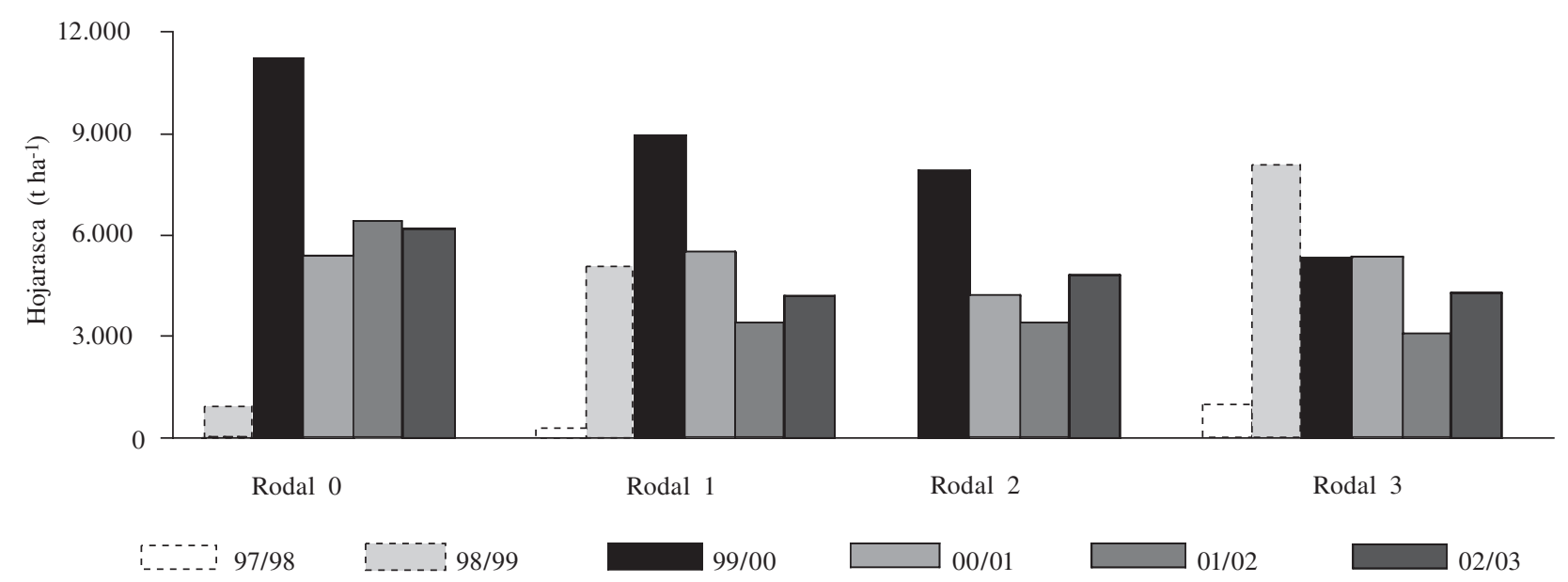

Figura 3. Cantidad anual de hojarasca aportada por distintos rodales en Eucalyptus nitens, entre 1997 y 2003 , predio Pichimaule, Fresia, X Región. Con línea punteada: cantidades estimadas.

Annual litter fall in an E. nitens plantation between 1997 and 2003, Fresia, 10 ${ }^{\text {th }}$ Region. Dot lines: estimated amounts.

La dispersión cuantitativa del mantillo, sin considerar ramas ni corteza (figura 5), mostró una amplia variabilidad en todos los rodales el año 2000 (coeficientes de variación entre 54 y 78\%). Esto se mantuvo el año 2003 para los rodales 0 y $1(C V=81$ a $85 \%)$; $\sin$ embargo, los rodales 2 y 3 presentaron un coeficiente de variación bastante menor (31 a 44\%), lo que es más bien casual.

Un factor importante en la dispersión espacial del mantillo fue la distribución de la caída de hojarasca asociada a su ubicación con respecto a los árboles (figura 6). Se observa que, especialmente el año 2000 (4 años de edad), cuando la plantación estuvo en proceso de cierre de sus copas, la mayor cantidad de hojarasca se concentró en la misma línea de árboles (posición A), disminuyendo hacia la posición $\mathrm{C}$ de entrelíneas. De esta manera, la cantidad de mantillo en la posición A fue significativamente mayor $(P<0,05)$ que en las posiciones $\mathrm{B}$ y $\mathrm{C}$, estas dos últimas sin diferenciarse $(P>0,05)$ entre sí. Esta tendencia se mantuvo en el año 2001, pero con el orden $\mathrm{A}=\mathrm{B}>\mathrm{C}(P<0,05)$. En el año 2002 las cantidades entre las posiciones $\mathrm{A}, \mathrm{B}$ y $\mathrm{C}$ no mostraron diferencias significativas $(P>0,05)$. Sin embargo, en el año 2003 volvió a destacar la acumulación en A $(\mathrm{A}>\mathrm{B}=\mathrm{C})(P<0,05)$, lo que indica que se mantuvo la mayor acumulación en la línea de los árboles.

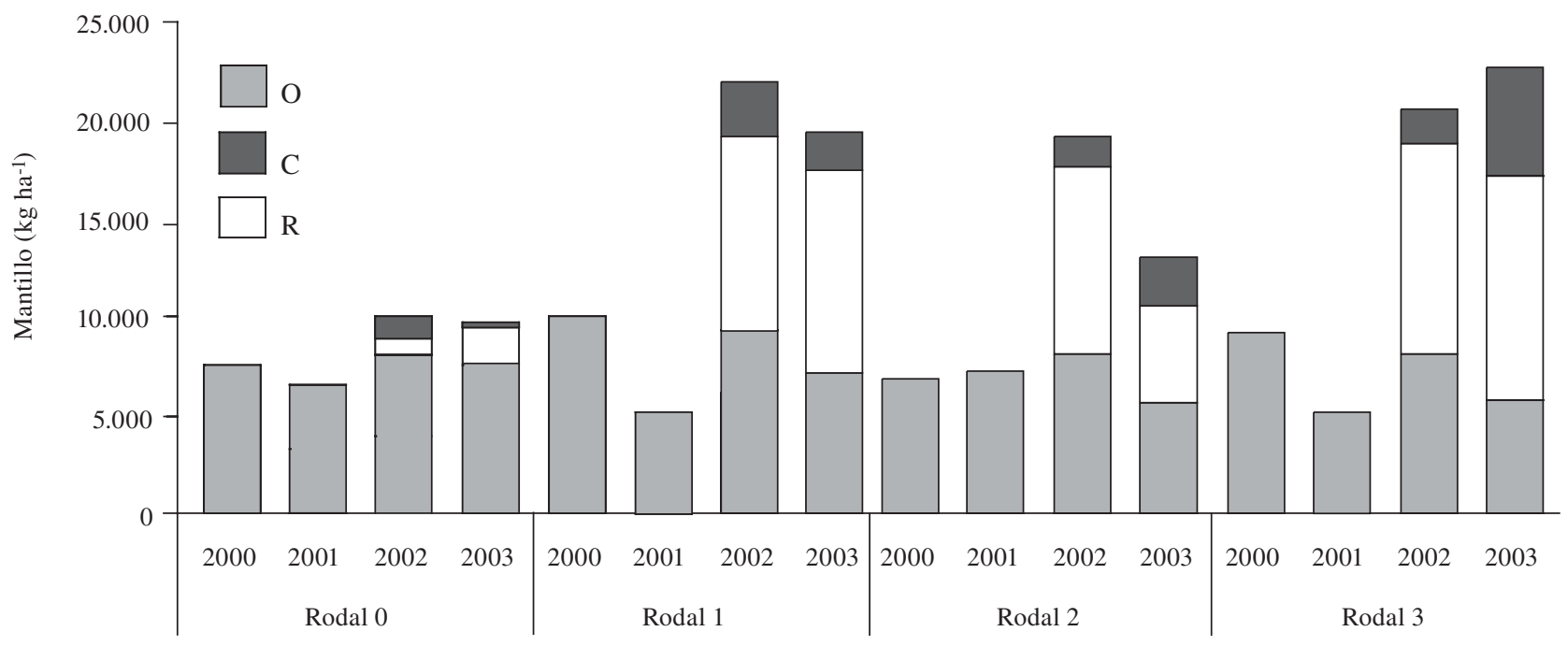

Figura 4. Cantidad de biomasa en el mantillo y composición pre y posraleo para distintos rodales en plantaciones de Eucalyptus nitens de 4 a 7 años de edad, predio Pichimaule, Fresia, X Región ( $\mathrm{O}=$ Horizonte orgánico, $\mathrm{C}=\mathrm{corteza}, \mathrm{R}=\mathrm{ramas})$.

Amount of biomass in the litter layer and quantitative effect of thinning in E. nitens, 4 to 7 years old, Fresia, $10^{\text {th }}$ Region $(\mathrm{O}=$ organic horizon, $\mathrm{C}=$ bark, $\mathrm{R}=$ branches $)$. 


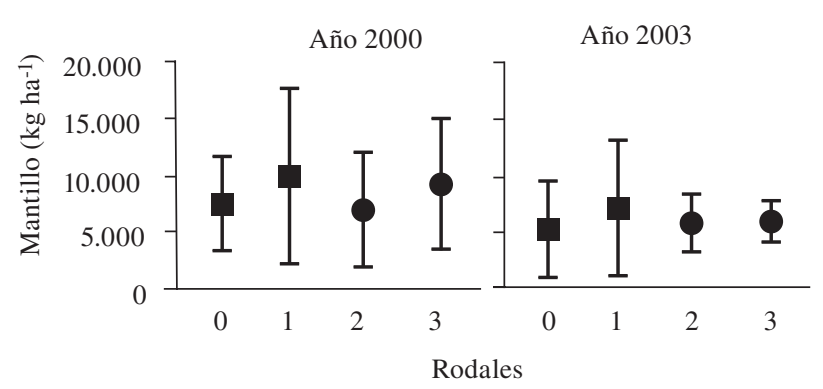

Figura 5. Dispersión del peso del mantillo (Oi y Oe) en plantaciones de Eucalyptus nitens de 4 y 7 años de edad para distintos rodales, predio Pichimaule, Fresia, X Región.

Dry weight variation of the litter layer (Oi and Oe) in $E$. nitens plantations, 4 to 7 years old, according to fertilization treatments, Fresia, $10^{\text {th }}$ Region.

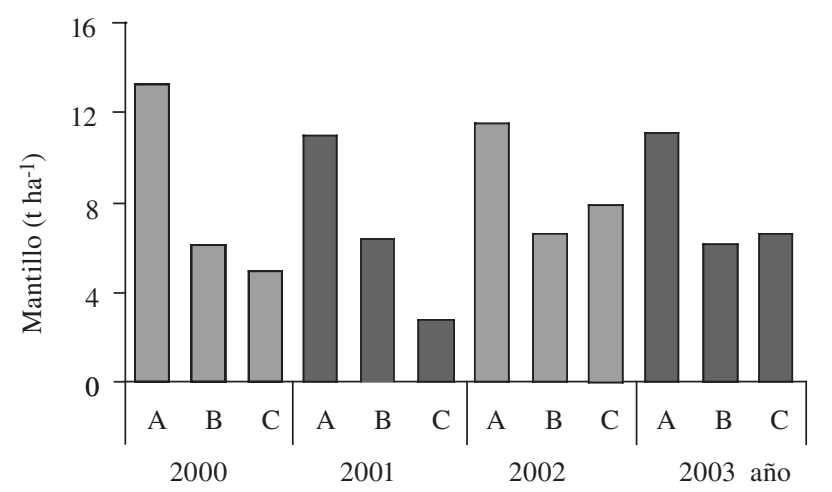

Figura 6. Variación del peso del mantillo entre las líneas de plantación para el rodal 0, en plantación de Eucalyptus nitens de 4 a 7 años de edad, predio Pichimaule, Fresia, X Región. A: línea de plantación, C: entre líneas; B: equidistante de A y C.

Dry weight variation of the litter layer between rows of trees in the stand $0, E$. nitens, 4 to 7 years old, Fresia, $10^{\text {th }}$ Region. $\mathrm{A}=$ under the trees, $\mathrm{C}=$ between tree lines, $\mathrm{B}=$ equidistant between $\mathrm{A}$ and $\mathrm{C}$.
La cantidad de mantillo (peso) se expresa generalmente a través de una función de su espesor. Sin embargo, si bien los resultados obtenidos en este estudio mostraron que entre tales variables hubo una asociación positiva, ésta fue fluctuante en el tiempo (figura 7). El año 2000 se obtuvo la relación más estrecha, pero luego decreció en los dos años siguientes. En todos los casos se encontró una alta dispersión del peso del mantillo para un determinado espesor de este horizonte.

El contenido nutritivo de la hojarasca (figura 8) mostró que en esta especie de Eucalyptus, como en otras del género, el elemento de mayor contenido es el calcio, seguido por el nitrógeno, el potasio y el fósforo. El nitrógeno, como era de esperar, mostró una gradación creciente: hojarasca $<$ Oi $<$ Oe. Los elementos calcio y fósforo también mostraron una tendencia al aumento del contenido desde la hojarasca hacia el mantillo (Oi), lo cual se vio modificado en el horizonte Oe para calcio en particular. En cambio, potasio presentó una clara tendencia a disminuir su contenido desde la hojarasca hacia el mantillo.

La cantidad de elementos nutritivos que aporta la hojarasca al suelo es el producto entre su contenido y la biomasa respectiva. Así el calcio fue el elemento cuantitativamente mayor, seguido por el nitrógeno, el potasio y luego el fósforo (cuadro 3). Lo anterior influyó en la acumulación de elementos en el mantillo, observándose la misma secuencia de la hojarasca $(\mathrm{Ca}>\mathrm{N}>\mathrm{K}>\mathrm{P})$ (figura 9). El rodal no raleado presentó cantidades menores de elementos nutritivos en el mantillo, a pesar de recibir mayor cantidad de hojarasca después del año 2001, ya que en los demás rodales se acumularon desechos del raleo y la poda.

La figura 10 muestra la proporcionalidad cuantitativa de los distintos elementos nutritivos por componente en el mantillo, precisando el efecto que tienen los desechos de raleo en ella. En el rodal 0 el subhorizonte Oi concen-

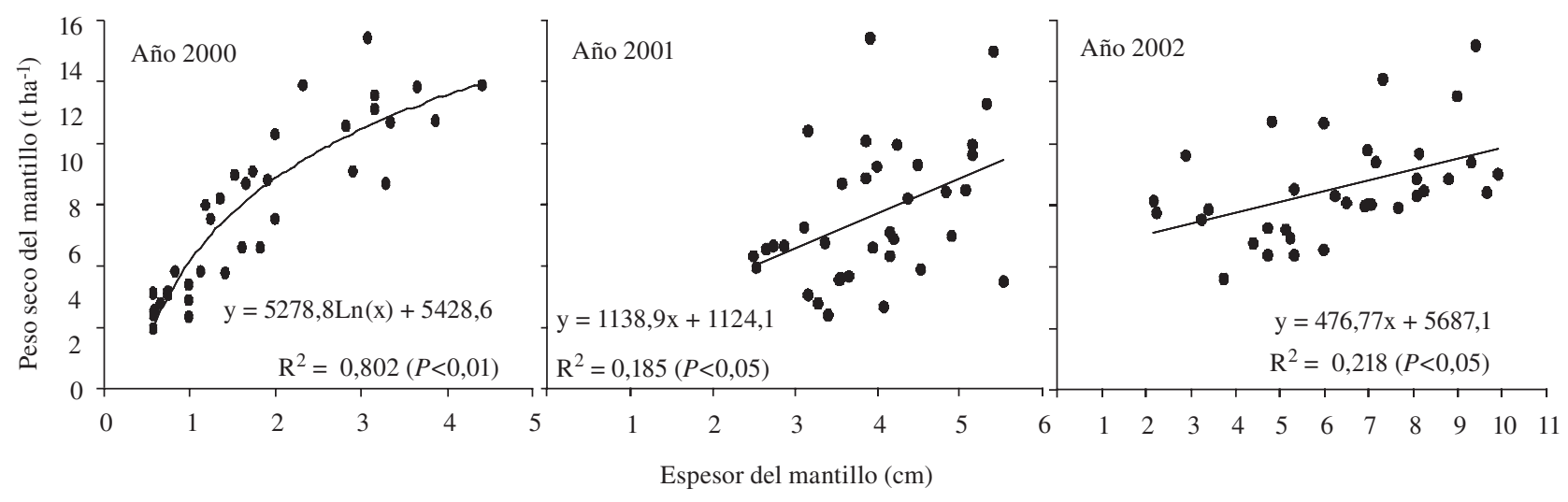

Figura 7. Peso del mantillo en Eucalyptus nitens de 4 y 5 años de edad en función de su espesor (rodal 0), predio Pichimaule, Fresia, X Región.

Correlation between dry weight and thickness of litter layer in E. nitens 4 and 5 years old (stand 0), Fresia, $10^{\text {th }}$ Region. 


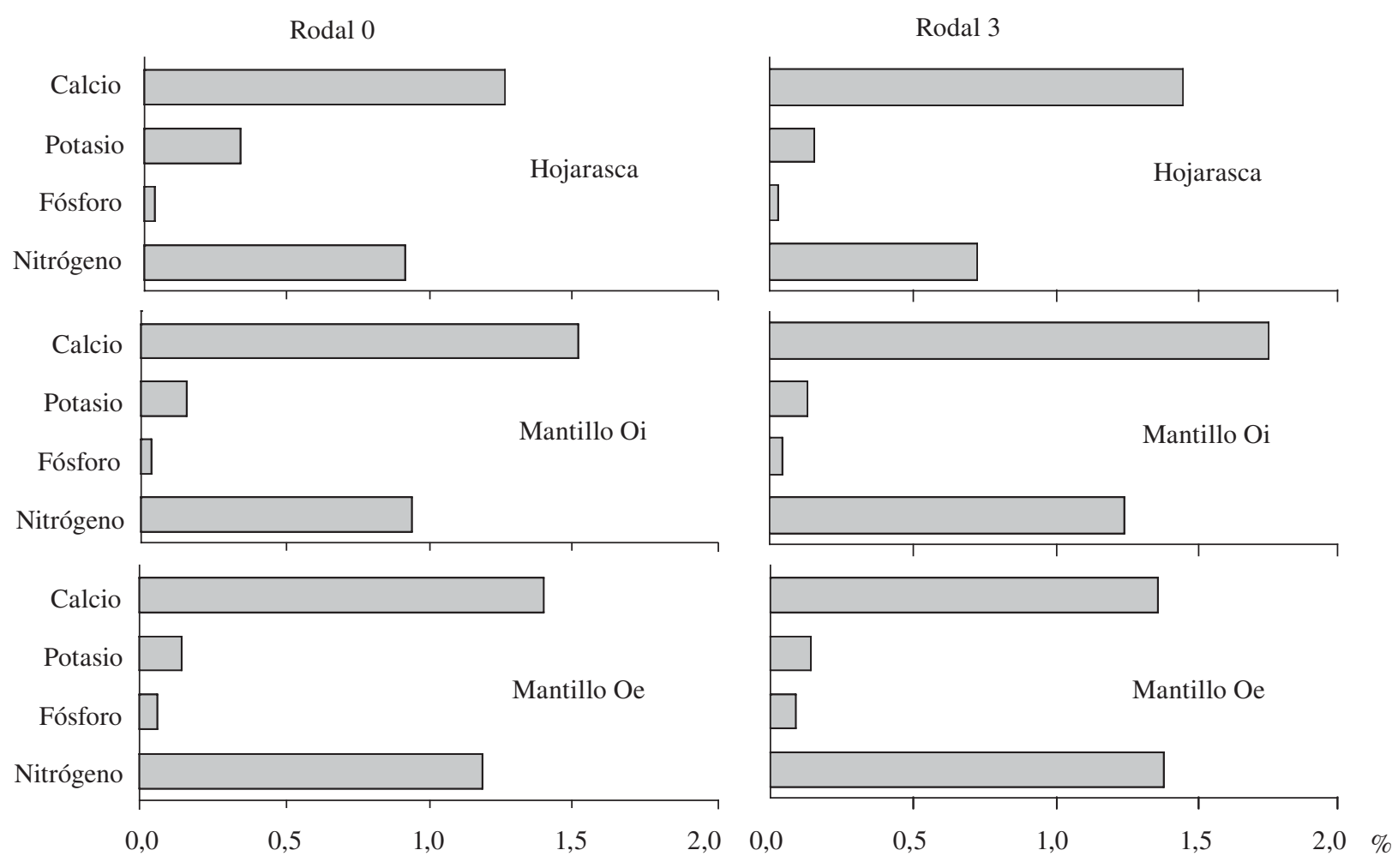

Figura 8. Contenido de elementos nutritivos en la hojarasca y mantillo (Oi y Oe) de Eucalyptus nitens de 7 años de edad, predio Pichimaule, Fresia, X Región.

Nutrient content in the litterfall and in the litter layer (Oi \& Oe) in an E. nitens plantation, at 7 years old, Fresia, $10^{\text {th }}$ Region.

tró la mayor proporción en una plantación sin raleo. En cambio, en los rodales con raleo la proporción en ramas y corteza ocupa una proporción muy importante, observándose entre ellos diferencias que representan la variación causada por la dispersión del material sobre el suelo (figura 5).

\section{DISCUSIÓN}

La caída de hojarasca estuvo influida por dos fenómenos naturales que modificaron los montos de desechos a través del tiempo. Por una parte, este proceso respondió a fluctuaciones climáticas estacionales, principalmente como respuesta al estrés hídrico estival (figura 1). Ello quedó reflejado en una mayor cantidad de hojas caídas en los meses más secos del verano, conducta que fue similar a la observada en otras y en la misma especie del género Eucalyptus en Australia (Bray y Gorham 1964, Moroni y Smethurst 2003). Sin embargo, la conducta anterior es válida sólo un año después del cierre del dosel de copas, ya que este último es un momento particular de alta competencia intraespecífica que ocasiona un desequilibrio o proceso de ajuste en la plantación, con menor acceso de luz a la copa baja y una mayor demanda por elementos nutritivos y agua por parte de la planta- ción. De hecho, la ocupación total del sitio induce a la abscisión de las hojas en una época más temprana del año (primavera), causando una caída extraordinaria en cuanto a la oportunidad y cantidad (figuras 2 y 3 ). Esto fue diferente a lo observado por Moroni y Smethurst (2003) en Australia, quienes no determinaron diferencias entre el momento del cierre de copas y los años sucesivos. Pero, en el presente estudio, el comportamiento cíclico relativamente constante de la caída de hojarasca se inicia un año después del cierre de copas, una vez logrado un nuevo equilibrio en la plantación.

Más adelante, la cantidad de hojarasca incluso se redujo por efecto de la intervención de raleo ejecutado a mitad de 2001 que causó una disminución de la cobertura del dosel y de la biomasa total en pie. En el raleo se extrajo el $47 \%$ de los árboles (cuadro 2), lo que causó una disminución de $25-40 \%$ en la caída de hojarasca en la temporada 2001-2002 (figura 2). Aun así el modelo de fluctuación estacional se mantuvo inalterado posteriormente y los rodales raleados se aproximan en forma paulatina al rodal no raleado en la temporada 2002-2003, a medida que el bosque recupera la ocupación total del sitio.

La caída de hojarasca tiene efectos en el piso del bosque, donde se acumula dando origen al mantillo. El mantillo constituye una capa de detritus, principalmente 


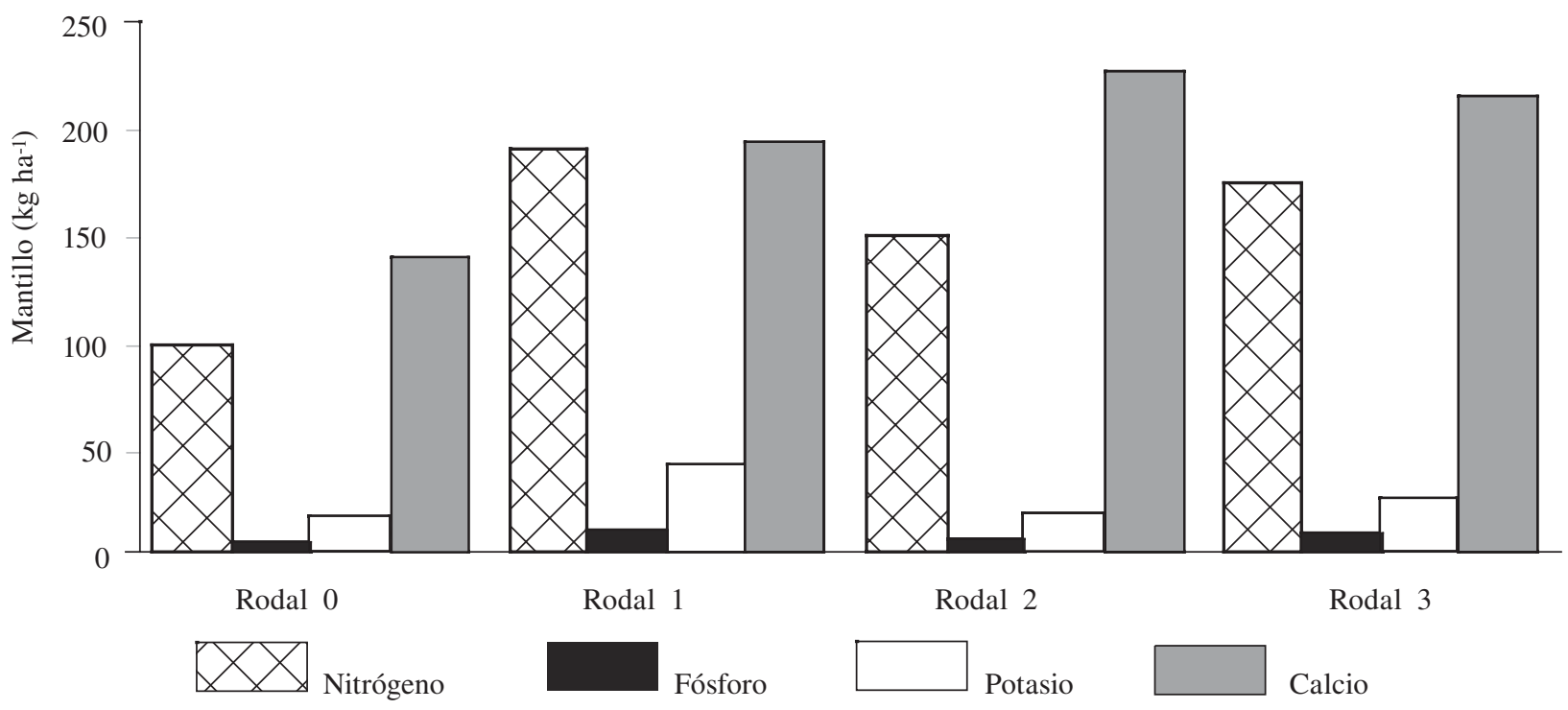

Figura 9. Cantidad de elementos nutritivos acumulados en el mantillo de Eucalyptus nitens en el año 2002 (6 años de edad), predio Pichimaule, Fresia, X Región.

Nutrient amount accumulated in the litter layer of E. nitens in 2002 (at 6 years old), Fresia, $10^{\text {th }}$ Region.

Cuadro 3. Cantidad de elementos nutritivos $\left(\mathrm{kg} \mathrm{ha}^{-1}\right)$ en la hojarasca de una plantación de Eucalyptus nitens de 4 a 7 años de edad, con distintos tratamientos de fertilización, predio Pichimaule, Fresia, X Región.

Amount of nutrients $\left(\mathrm{kg} \mathrm{ha}^{-1}\right)$ in litter fall of an E. nitens plantation, between 4-7 years old according to treatment, Fresia, $10^{\text {th }}$ Region.

\begin{tabular}{|c|c|c|c|c|c|}
\hline \multirow[b]{2}{*}{ Rodal } & \multirow{2}{*}{$\begin{array}{c}\text { Elemento } \\
\left(\mathrm{kg} \mathrm{ha}^{-1}\right)\end{array}$} & \multicolumn{4}{|c|}{ Período anual (edad en años) } \\
\hline & & $\begin{array}{c}99 / 00 \\
(3-4)\end{array}$ & $\begin{array}{c}00 / 01 \\
(4-5)\end{array}$ & $\begin{array}{l}01 / 02 \\
(5-6)\end{array}$ & $\begin{array}{c}02 / 03 \\
(6-7)\end{array}$ \\
\hline \multirow{4}{*}{0} & Nitrógeno & 49 & 42 & 52 & 42 \\
\hline & Fósforo & 2 & 1 & 2 & 2 \\
\hline & Potasio & 43 & 15 & 25 & 22 \\
\hline & Calcio & 151 & 75 & 87 & 60 \\
\hline \multirow{4}{*}{1} & Nitrógeno & 50 & 47 & 30 & 27 \\
\hline & Fósforo & 4 & 2 & 1 & 1 \\
\hline & Potasio & 39 & 22 & 11 & 12 \\
\hline & Calcio & 94 & 60 & 31 & 36 \\
\hline \multirow{4}{*}{2} & Nitrógeno & 40 & 30 & 23 & 26 \\
\hline & Fósforo & 2 & 1 & 1 & 1 \\
\hline & Potasio & 22 & 11 & 7 & 10 \\
\hline & Calcio & 86 & 61 & 47 & 53 \\
\hline \multirow{4}{*}{3} & Nitrógeno & 29 & 35 & 20 & 22 \\
\hline & Fósforo & 2 & 1 & 1 & 1 \\
\hline & Potasio & 15 & 13 & 5 & 7 \\
\hline & Calcio & 68 & 84 & 47 & 50 \\
\hline
\end{tabular}
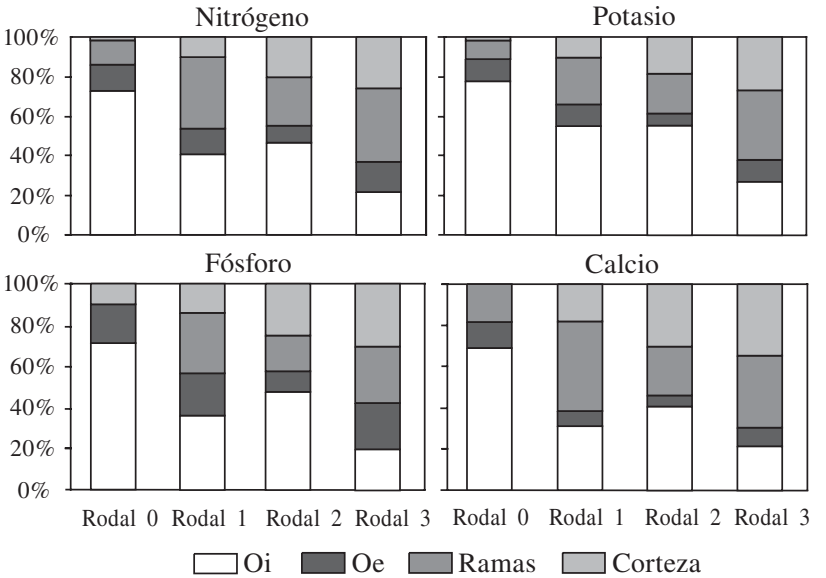

Figura 10. Distribución porcentual de elementos nutritivos en el mantillo de E. nitens de 6 años de edad, con diferentes tratamientos, Fresia, X Región. Rodal 0, sin raleo.

Nutrient distribution (\%) in the litter layer of E. nitens 6 years old, according to treatments, Fresia, $10^{\text {th }}$ Region. Stand 0, not thinned.

hojas, que cubre el suelo protegiéndolo contra la erosividad de la lluvia y los cambios bruscos de temperatura, disminuyendo la evapotranspiración y manteniendo el suelo con mayor humedad (Schlatter et al. 2003). En estas plantaciones el mantillo presentó una forma de humus mull, tipo F (Arbeitsgruppe Boden 1994), por su morfología Oi (Oe), es decir, con escasa acumulación en el horizonte Oe y ausencia del horizonte $\mathrm{Oa}$, y por la profundidad de los horizontes $\mathrm{A}$ y $\mathrm{AB}$, mayor a $10 \mathrm{~cm}$ (17 cm ambos), en el suelo mineral. La morfología y la cantidad de biomasa presente en el horizonte $\mathrm{O}$ (sin con- 
siderar ramas y corteza producto del raleo) son indicadores de una descomposición rápida de este residuo, ya que no se observa una acumulación creciente en ninguno de los rodales (figura 4 ).

La similitud de los promedios de la cantidad de mantillo desde el año 2000 hasta 2003 (figura 5) indica que la descomposición de esta materia orgánica en las plantaciones de E. nitens es muy rápida, permitiendo así que la mineralización de los elementos nutritivos sea ágil. Geldres (2005ab) estimó para estas mismas plantaciones que los macroelementos aquí estudiados (N, P, K, Ca) presentaban una retención generalmente no superior a 2,5 años en el mantillo. El ciclo biogeoquímico en el ecosistema puede considerarse, en consecuencia, como fluido, así también, por este intermedio, el abastecimiento nutritivo de los árboles.

El horizonte $\mathrm{O}$ mostró la variabilidad natural (figura 5 ) en su peso seco entre rodales y entre años, además de un eventual efecto de error por el método (puntos de recolección distintos), lo cual influyó en la variabilidad observada, pero no en las tendencias de las medias. La mayor acumulación de mantillo en el horizonte $\mathrm{O}$ de los rodales 1 y 3 en el año 2000 con respecto al 2001 (figura 6) se explica, en gran parte, como consecuencia de la mayor caída de hojarasca en el período previo inmediato, por cierre de copas del dosel, como se observó en visitas anteriores a la plantación. Ambos rodales cerraron sus copas antes que los otros dos y, por lo tanto, el proceso de caída de hojas se adelantó en ellos (figura 3).

La distribución espacial del mantillo conservó una variación asociada a la posición con respecto a los árboles. En todos los años observados la posición A (figura 6), ubicada directamente bajo las copas en las líneas de plantación, acumuló más desechos que las posiciones más alejadas de los fustes, diferencia que gradualmente fue menor con los años. Las faenas de raleo, a mediados del año 2001, dispersaron y reubicaron parte del material, lo que explica la distribución más irregular determinada a los 6 años de edad. Sin embargo, a los 7 años de edad, 2 años después del raleo, nuevamente la posición A se diferencia de los puntos entre hileras, con una mayor acumulación de mantillo. Esto último depende, sin embargo, del espaciamiento entre los árboles. En esta plantación las hileras estuvieron separadas $4 \mathrm{~m}$ entre sí; sin embargo, en plantaciones con hileras de menor distanciamiento probablemente estas diferencias desaparecen muy pronto después del cierre de copas (Moroni y Smethurst 2003).

La cantidad de mantillo estimada a partir del espesor, según los resultados del presente estudio, indica que ese parámetro es poco confiable para su cuantificación, por la gran dispersión observada. De hecho, según la densidad del material componente del mantillo, el peso puede ser muy distinto para un mismo espesor (figura 7). Por eso, es recomendable determinar el peso del mantillo directamente, a través de muestras de bio- masa de los desechos acumulados. De todas maneras, el espesor del mantillo complementa la adecuada caracterización de su morfología para definir la forma de humus (Schlatter et al. 2003). La forma de humus permite inferir sobre los aspectos cualitativos de éste, como también con respecto a la calidad del proceso de descomposición.

Por efectos del raleo, el cual fue efectuado después de la recolección de mantillo a mediados del año 2001, se incorporó una significativa masa de ramas y corteza al piso, que se detectó en los muestreos de 2002 y 2003, y que aumentó su ponderación cuantitativa con respecto al rodal sin raleo (figura 4 ).

En la secuencia de componentes hojarasca-Oi-Oe, Eucalyptus nitens mostró contenidos y cantidades de elementos nutritivos con la tendencia: calcio $>$ nitrógeno $>$ potasio $>>$ fósforo. Este orden difiere de los observado en Pinus radiata D. Don, por ejemplo, donde la gradación cuantitativa es nitrógeno $>$ potasio $>$ calcio $>>$ fósforo, y donde, además, las cantidades por unidad de biomasa son en general menores (Gerding 1991). El alto contenido de calcio, sin duda, es uno de los factores más importantes en la rápida descomposición del mantillo en los Eucalyptus sp., ya que regula el $\mathrm{pH}$, es decir, permite un mejor equilibrio entre los elementos nutritivos (Schlatter y Otero 1995) y favorece la actividad microbiológica (Schroeder 1984).

Los contenidos del nitrógeno y del fósforo (figura 8), en general similares o más bajos que los indicados por Moroni y Smethurst (2003) en Tasmania, aumentaron algo en la secuencia: hojarasca < Oi < Oe, como es de esperar, ya que en el proceso de descomposición estos elementos son retenidos por los microorganismos o compuestos orgánicos a diferencia del carbono, el cual se pierde en su mayor parte por la respiración de la actividad biológica (Will 1967). La variación del calcio puede tener el mismo origen, pero su disminución en Oe puede ser causa de una mayor lixiviación o consumo por los organismos del suelo. La disminución del contenido de potasio en el mantillo, comparado con la hojarasca, se explica, porque es un elemento muy móvil en la condición de componente de los tejidos orgánicos, especialmente en climas lluviosos (Schlatter 1988, Gerding 1991).

Las cantidades de elementos nutritivos acumuladas en el mantillo son lideradas por el calcio, seguido muy de cerca por el nitrógeno (figura 9). Las reservas que representan estos elementos son muy importantes por su función como fuente nutritiva para la fauna y flora heterótrofa del suelo y, por su intermedio, para el abastecimiento nutritivo de los árboles que puede reducir considerablemente los costos para mantener la fertilidad del suelo en este tipo de cultivo forestal. El raleo y la poda contribuyen a aumentar temporalmente estas cantidades y favorecen el desarrollo de los árboles remanentes. Por eso es importante dejar estos desechos silviculturales en 
el mismo sitio, dispersos en el lugar de cosecha, como aportes al balance de los elementos nutritivos y así contribuir a la sustentabilidad de una plantación forestal (figura 10). La proporción de elementos nutritivos que retornan los desechos del raleo y la poda es considerable, aumentando según la productividad del sitio y la intensidad del raleo.

\section{CONCLUSIONES}

La máxima caída de hojarasca se alcanzó al cierre de copas de la plantación y en los años siguientes disminuyó, manteniendo una tasa anual constante, la que en caso de raleo fue menor. La mayor proporción de esta caída ocurrió en verano (diciembre a febrero) como respuesta a un estrés hídrico, con excepción del momento del cierre de copas, que fue cuantitativamente decisivo en este proceso. A través de la hojarasca regresaron al suelo cantidades significativas de elementos nutritivos para el abastecimiento de la plantación.

Hasta los 5 años se observó mayor cantidad de mantillo en las líneas de árboles y una disminución gradual de la cantidad hacia las posiciones más alejadas entre líneas. Posteriormente, y directo bajo los árboles, se mantuvo una mayor cantidad de mantillo, pero en el resto de la superficie tendió a una homogenización. La retención de la corteza y las ramas en el piso del bosque, luego del raleo tuvo efectos significativos en las cantidades de elementos nutritivos que permanecieron en el sitio, en especial, con respecto al calcio.

La forma de humus mull y la cantidad de materia orgánica y de elementos nutritivos en el mantillo permiten inferir sobre una rápida descomposición de los desechos de la plantación de E. nitens, lo que debiera dar origen a ciclos muy dinámicos de los elementos nutritivos, favorables para el abastecimiento de los árboles.

Entre el flujo de la hojarasca y el mantillo existió una tendencia a la retención del nitrógeno, del fósforo y del calcio durante el proceso de descomposición, al contrario del potasio, que es lixiviado desde la materia orgánica, presentando, en consecuencia, una tendencia a disminuir su contenido a medida que la materia orgánica se descompone.

El espesor del mantillo no fue un parámetro confiable para la estimación cuantitativa del peso de éste, por la alta variación de su densidad. Para lograr datos precisos debe determinarse el peso seco, el cual para su interpretación cualitativa debe ser complementado con la caracterización morfológica del horizonte orgánico $(\mathrm{O})$.

\section{AGRADECIMIENTOS}

Financiado por FONDECYT 1010174 y Universidad Austral de Chile/DID-S-200024.

\section{REFERENCIAS}

Anderson JM. 1991. The effects of climate change on decomposition processes in grassland and coniferous forests. Ecological Applications 1:326-347.

Aparicio J. 2001. Rendimiento y Biomasa de Eucalyptus nitens con alternativas nutricionales para una silvicultura sustentable en un suelo rojo arcilloso. Tesis Magíster en Ciencias. Valdivia, Chile. Facultad de Ciencias Forestales, Universidad Austral de Chile. 234 p.

Arbeitsgruppe Boden. 1994. Bodenkundliche Kartieranleitung. Hannover, Alemania. Bundesanstalt für Geowissenschaften-Hannover, $4^{\text {a }}$ edición. 392 p.

Belmar L. 2003. Nitrógeno y calcio en la biomasa aérea de plantaciones de cinco años de Eucalyptus nitens (Dean et Maiden) Maiden con distintas fertilizaciones (comuna de Fresia, X Región). Tesis Ingeniero Forestal. Valdivia, Chile. Facultad de Ciencias Forestales, Universidad Austral de Chile. 40 p.

Blair JM, RW Parmelee, MH Beare. 1990. Decay rates, nitrogen fluxes and decomposer communities of single and mixed species foliar litter. Ecology 71:1976-1985.

Bonomelli C, I Peña, D Suárez. 2002. Contribución de la hojas en el proceso de reciclaje de $\mathrm{N}$ y $\mathrm{P}$ en Eucalyptus sp. Bosque 23 (1):61-77.

Bray R, E Gorham. 1964. Litter production in forests of the World. Advances in Ecological Research 2:101-157.

CIREN (Centro de Información de Recursos Naturales, CL). Estudio agrológico X Región. 2001. Descripciones de suelos, materiales y símbolos. Tomo I. Santiago, Chile. Centro de Investigación de Recursos Naturales. 199 p.

Donoso C. 1992. Ecología Forestal; el bosque y su medio ambiente. $5^{\text {a }}$ edición, Santiago, Chile. Universitaria. $369 \mathrm{p}$.

Geldres E. 2005a. Dinámica de fósforo y calcio en Eucalyptus nitens de 4 a 7 años en un suelo rojo arcilloso. Tesis Magíster en Recursos Forestales. Valdivia, Chile. Facultad de Ciencias Forestales, Universidad Austral de Chile. 89 p.

Geldres E. 2005b. Exportación de nitrógeno y potasio con la cosecha de Eucalyptus nitens. Tesis Magíster en Gestión Ambiental. Valdivia, Chile. Facultad de Ciencias Forestales, Universidad Austral de Chile. 46 p.

Gerding V. 1991. Pinus radiata-Plantagen in Zentralchile: Standortsfaktoren der Produktivität und Nährstoffverteilung in Beständen. Tesis Doctorado. Göttingen, Alemania. Forstwissenschftlicher Fachbereich. Universidad GeorgAugust-Göttingen. 182 p.

Gerding V, JE Schlatter, C Saavedra. 2002. Biomasa de plantaciones de Eucalyptus nitens de 5 años en un suelo rojo arcilloso con fertilización, comuna de Fresia, X Región. In Primer Congreso Chileno de Ciencias Forestales. Universidad de Chile. Santiago, Chile. 8 p.

González C. 2003. Fósforo y azufre en la biomasa aérea de plantaciones de cinco años de Eucalyptus nitens (Dean et Maiden) Maiden con diferentes fertilizaciones. Tesis Ingeniero Forestal. Valdivia, Chile. Facultad de Ciencias Forestales, Universidad Austral de Chile. 40 p.

Hartung J, B Elpelt, KH Klösener. 2002. Statistik, Lehr- und Handbuch der angewandten Statistik. 13. ed., Oldenburg Verlag. München, Alemania. 975 p. 
Leiva F. 2000. Efectos de una roca fosfórica y una cal como tratamientos base en una plantación de Eucalyptus nitens (Maiden) en un suelo rojo arcilloso de la comuna de Fresia, X Región. Tesis Ingeniero Forestal. Valdivia, Chile. Facultad de Ciencias Forestales, Universidad Austral de Chile. 82 p.

Moroni MT, PJ Smethurst. 2003. Litterfall, nitrogen and phosphorous fluxes in two Tasmanian Eucalyptus nitens plantations. Tasforests 14:53-63.

Schlatter JE. 1988. Información sobre el elemento potasio en el sector forestal nacional. Boletín $\mathrm{N}^{\circ}$ 8, Sociedad Chilena de la Ciencia del Suelo. p. 123-143.

Schlatter JE, V Gerding, H Huber. 1995. Sistema de ordenamiento de la tierra; herramienta para la planificación forestal, aplicado a la X Región. Universidad Austral de Chile. Valdivia, Chile. 93 p.

Schlatter JE, L Otero. 1995. Efecto de Pinus radiata sobre las características químico-nutritivas del suelo mineral superficial. Bosque 16 (1):29-46.
Schlatter J, R Grez, V Gerding. 2003. Manual para el reconocimiento de suelos. Universidad Austral de Chile. Valdivia, Chile. 114 p.

Schroeder D. 1984. Bodenkunde in Stichworten. Ferdinand Hirt Verlag. Zug, Suiza. 160 p.

Staub J. 2000. Evaluación de una fertilización basada en fósforo al inicio de una plantación de Eucaliptus nitens (Deane y Maiden) Maiden en un suelo rojo arcilloso de la X Región. Tesis Ingeniero Forestal. Valdivia, Chile. Facultad de Ciencias Forestales, Universidad Austral de Chile. 94 p.

Swift M J, OW Heal, JM Anderson. 1979. Decomposition in terrestrial ecosystems. In Studies in Ecology $\mathrm{N}^{\circ}$ 5, University of California Press. Berkeley, U.S.A.

Vitousek PM, DR Turner, WJ Parton, RL Sanford. 1994. Litter decomposition on the Mauna Loa environmental matrix, Hawaii: patterns, mechanisms, and models. Ecology 72:418-429.

Will GM. 1967. Decomposition of Pinus radiata litter on the forest floor. Part I. Changes in dry matter and nutrient content. New Zealand Journal of Science 10 (4):1030-1044. 